\title{
CSR Based on Game Theory
}

\author{
Weixiao Zhu, Hongyan Li \\ College of Management, Shanghai University of Engineering Science, Shanghai, China. \\ Email: zhuweixiao12@163.com, lihongyan580@sohu.com
}

Received October $10^{\text {th }}, 2103$; revised November $10^{\text {th }}, 2013$; accepted November $15^{\text {th }}, 2013$

Copyright (C) 2013 Weixiao Zhu, Hongyan Li. This is an open access article distributed under the Creative Commons Attribution License, which permits unrestricted use, distribution, and reproduction in any medium, provided the original work is properly cited.

\begin{abstract}
In recent years, Corporate Social Responsibility (CSR) has gradually become a hot topic with the public's increasing concern. In this study, authors briefly introduce some reviews and concepts of CSR, and then combine the idea of Game Theory and Matrix linear equations method to conduct an empirical analysis between enterprises with enterprises and enterprises with government. By analysis, we find that it is very important for the development of enterprises in these two parts: the fulfillment of social responsibility and the supervision of government. Finally, the authors also put forward some suggestions to the fulfillment of CSR and hope to improve the poor sense of presence.
\end{abstract}

Keywords: Corporate Social Responsibility; Game Theory; Linear Equations Method

\section{Introduction}

Corporate Social Responsibility (CSR) doesn’t appear very long, but it has caused an extensive attention in the academic and practical field, which makes domestic and foreign scholars study diligent. This fully shows that CSR is of significant value in researching. By studying the existed literature, we find that the formers' research from the perspective of game theory is not very much and also not very comprehensive. This article intends to introduce game theory into the study of CSR, and it uses the relevant content to analyze the relationship between enterprises with other enterprises and enterprises with government in the process of fulfilling CSR. We can find the intrinsic link among them, then deeply excavate factors that affect enterprise to fulfill the social responsibilities. We find the underlying reasons for the low level of CSR in China. Eventually we can propose the appropriate measures. It is of great theoretical significance in China's enterprises actively fulfilling the social responsibility, building a harmonious society and implementing the concept of scientific development.

From the point of enterprise's own survival and development, we introduce the fulfillment of CSR into the framework of game theory. The concept of CSR is wide, whether it is implemented or not and the extent of it all are influenced by various stakeholders in making decisions. Whether the government supervises CSR or not and the intensity of it, as well as whether the staff is loyal to the enterprise or not, whether the competitor fulfills its social responsibility or not and whether the consumer buys the products of company or not, all have direct impacts on the fulfillment of CSR.

\section{Literature Review}

\subsection{CSR}

Since 1924, Oliver Sheldon proposed the concept of CSR for the first time; it has been widely concerned by all sectors of society. He believed that enterprises do not only have the economic obligations and legalities, but also assume the social responsibility which beyond these obligations. Y. Li et al. [1] proposed "the model of three levels and four cores”, which gave a more complete, rigorous and dynamic definition of CSR. Namely, "in a particular period of social development, the enterprise should assume the economic, regulatory, ethical, voluntary charitable and other related responsibilities for its stakeholders.” D. Lu [2] thought the connotation of CSR was that "enterprise is a commercial organization and its goal is to maximize shareholders' interests, CSR assumes the responsibility of preserving and enhancing social welfare value while in the pursuit of maximizing shareholders' interests for the purpose.

In the aspect of method and model, L. Zhang et al. [3] used Analytic Hierarchy Process study CSR; F. Wei et al. [4] used Solow model; S. Mai et al. [5] used TOPSIS and so on. But the research of it by game theory is less. This paper tries to use the game theory to analyze the impor- 
tance of fulfilling CSR.

\subsection{Game Theory}

Game theory studies how rational people choose the tactics. Nash established the classic story of game theory which called "Prisoner's Dilemma", this described the establishment of non-cooperative game and equilibrium solution, this is why it called "Nash equilibrium". In this story, the two prisoners chose to admit committing murder, then two people all won the intermediate results of pronounce judgment. If one of them confessed the murder, and the other don't, then the one who admitted would be commuted a sentence, and another would be sentenced to ten years in prison.

In the game, if all participants have a dominant strategy, it proves that the game will reach equilibrium on the basis of the dominant strategy of all participants; this equilibrium is called dominant strategy equilibrium. The prisoner's dilemma above mentioned "prisoner A confesses, B confesses” is the dominant strategy equilibrium.

Prisoner's dilemma reflects a profound problem: the conflicts between individual rationality and group rationality. Then you will need to introduce Nash equilibrium. Nash equilibrium points that each participant in the game convinces that in a given case of other participants' chosen strategy, the participant chooses the optimal strategy in response to the opponent's strategy.

\section{The Analysis of CSR That Based on Game Theory}

\subsection{Prisoner's Dilemma of Two Enterprises with No Government Involvement}

The assumptions of basic model:

First: there are only Companies 1 and 2 in the market, Companies 1 and 2 are "rational economic man", they are in pursuit of profit maximization, excluding emotional factors;

Second: Companies 1 and 2 all faced incomplete and asymmetric information, namely is any one firm does not know another firm's strategy in choosing;

Third: in the market, Companies 1 and 2 have two choices: bear the social responsibility or not;

Fourth: once Companies 1 and 2 make a decision, it can not be changed. Therefore, we establish a payoff matrix shown in the following Table 1, the first number is the benefit of selecting Company 1 , the second number is the benefit of selecting Company 2 .

If $a<c$ and $b<d$, the benefits that companies not fulfilling their CSR is greater than that companies do, then two companies trap into Prisoner's Dilemma. They achieves Nash equilibrium in $(d, d)$ (each party of game gets the equilibrium in the strategy of game), namely both of them do not fulfill their social responsibility.
Table 1. The payoff matrix of the two companies.

\begin{tabular}{cccc}
\hline & \multicolumn{2}{c}{ Company 2 } \\
\cline { 3 - 4 } & & Fulfill & Not fulfilling \\
\hline \multirow{2}{*}{ Company 1 } & Fulfill & $(a, a)$ & $(b, c)$ \\
& Not fulfilling & $(c, b)$ & $(d, d)$ \\
\hline
\end{tabular}

Company's behavior will produce external effects, including external economies and external diseconomies. External influence can be here and there, various activities of companies will produce external influence. The effect produced by certain acts of CSR has the nature of public product, and can not be ruled out by the circumstance that other enterprises, consumers or organizations share their benefits without paying the cost, which is called "free rider". Thus we say, "It is the public goods" nature of CSR activities that restricts companies from undertaking their social responsibilities actively."

\subsection{The Analysis of Tactics' Selection between Government and Company in the Disposable Game}

The assumptions of basic model:

First: there are two participants in the model: company and government;

Second: company is regarded as a "rational economic man”, in pursuit of profit maximization; there is no inherent enthusiasm in undertaking social responsibility. Government is regarded as a representative on behalf of interests of the public. On the one hand government can promote economic growth, on the other hand, it can bear pressure from the public, and be required to supervise companies to undertake CSR, such as improve the environment in order to boost the quality of life;

Third: As shown in Table 2, companies are facing with two choice: "bear social responsibility" or "not bearing” during the game. $M$ is the required cost of company's undertaking CSR, assuming that if company undertakes CSR, the government will get profits from this part; $N$ is the cost of the government supervision, $F$ is the fine, and $F>N$; the first number is the benefit of selecting the government, the second number is the benefit of selecting the company.

We assume $p$ is the probability of government supervision, $q$ is the probability of company's undertaking CSR, for a given " $q$ ", the expected benefit of government supervision $(p=1)$ or without supervision $(p=0)$ are respectively as follows:

If the government supervising, its expected benefit is $W_{1}(1, q)=(M-N) \cdot q+(M+F-N) \cdot(1-q)$.

If the government does not supervise, its expected benefit is $W_{2}(0, q)=M \cdot q+0 \cdot(1-q)$. 
Table 2. The payoff matrix with the government's involvement.

\begin{tabular}{cccc}
\hline & \multicolumn{2}{c}{ Company } \\
\cline { 3 - 4 } Government & Fulfill $(q)$ & Not fulfilling $(1-q)$ \\
\hline & $\begin{array}{c}\text { Supervise }(p) \\
\text { Without } \\
\text { supervision } \\
(1-p)\end{array}$ & $(M-N,-M)$ & $(M+F-N,-F-M)$ \\
\hline
\end{tabular}

If the benefit of whether the government supervises or not is same, namely is $W_{1}(1, q)=W_{2}(0, q)$, so

$q^{*}=(M+F-N) /(M+F)$. If the probability of company's undertaking social responsibility is greater than $(M+F-N) /(M+F)$, the government will choose not to supervise; if the probability is less than

$(M+F-N) /(M+F)$, the government will choose to supervise; if the probability is exactly equal to $(M+F-N) /(M+F)$, the government can randomly choose whether supervise or not.

For a given " $p$ ”, the expected benefits of company's undertaking social responsibility $(q=1)$ or not undertaking $(q=0)$ are as follows:

If companies chose to undertake, its expected benefit is $W_{3}(p, 1)=-M \cdot p+(-M) \cdot(1-p)$.

If companies chose not to undertake, its expected benefit is $W_{4}(p, 0)=(-F-M) \cdot p+0 \cdot(1-p)$.

If the benefit of whether the company undertakes or not is same, namely is $W_{3}(p, 1)=W_{4}(p, 0)$, so

$p^{*}=M /(M+F)$. If the probability of government supervision is less than $M /(M+F)$, the company will choose not to undertake social responsibility; if the probability of government's supervision is greater than $M /(M+F)$, then the company will choose to undertake social responsibility; if the probability is exactly equal to $M /(M+F)$, the company can randomly select whether undertake or not. .

Therefore, the Nash equilibrium of mixed strategy is: $\left(p^{*}, q^{*}\right)=(M /(M+F),(M+F-N) /(M+F))$,

namely the government is willing to supervise by the probability of $M /(M+F)$, and company willing to undertake the social responsibility by the probability of $(M+F-N) /(M+F)$. Through analyzing Nash equilibrium, we find that game equilibrium solutions are closely related with the cost of supervision, management and fine. Visibly, the more the cost of supervision, the lower the probability of company's undertaking social responsibility. Because company knows the government will carry out a cost-effectiveness analysis rationally and reduce the strength of supervision. The higher the cost of management, the greater probability of company's undertaking social responsibility. If companies do not take social responsibility they will bring bad social influence, because they may be afraid of affecting reputation and taking more social responsibility. The higher the fines, the greater probability of company's undertaking social responsibility. Because the government thinks that companies would be afraid of large fines, and be willing to take social responsibility. So, in the disposable game, companies and government choose not to supervise or undertaking in a large extent, the two sides do not achieve their optimal choice or profits maximization as a whole. So Prisoner's dilemma is still existed. The best way to solve Prisoner's dilemma is gaming repeatedly.

\section{Empirical Study}

\subsection{Two Companies' Prisoner's Dilemma with No Government Involvement}

There are only Company 1 and Company 2 in the market, and the payoff matrix of whether fulfill the responsibility they face as follows (see Table 3):

Matrix $G=\left(S, D, A_{1}\right), S$ is the strategy of Company $1, D$ is the strategy of Company $2, A_{1}$ is the pay of the armor. $A_{1}=\left[\begin{array}{ll}5 & 1 \\ 9 & 3\end{array}\right]$, taking a minimum from each row and a maximum from each line, we can find there exists the saddle point $\left(S_{i}{ }^{*}, d_{j}{ }^{*}\right)=\left(S_{2}, d_{2}\right)=(3,3)$, the game value $V_{G} *=3$.

From Table 3, we know that the benefit that company's not fulfilling the social responsibility is greater than they do, so the two companies trap into Prisoner's Dilemma. They reached Nash equilibrium in $(3,3)$, namely both of them do not undertake their social responsibilities.

\subsection{The Analysis of Tactics' Selection between Government and Company in the Disposable Game}

We have surveyed a company and find that if company undertakes CSR, then they should pay ten units, the cost of government's supervision is two units, the cost of fines is three units. The payoff matrix with the government's involvement is as follows (see Table 4):

Matrix $G=\left(S, D, A_{2}\right), A_{2}=\left[\begin{array}{cc}8 & 11 \\ 10 & 0\end{array}\right]$, taking a minimum from each row and a maximum from each line. We can find there is no saddle point, namely, there is no solution in the sense of pure strategy. At this time, we introduce $2 \times 2$ matrix linear equations.

Suppose that the mixed strategy of government is $(x, 1-x), x \in[0,1]$; the mixed strategy of company is $(y, 1-y), \quad y \in[0,1]$; the equations are as follows:

$$
\begin{array}{ll}
8 x+10(1-x)=v & 8 y+11(1-y)=v \\
11 x+0(1-x)=v & 10 y+0(1-y)=v
\end{array}
$$


Table 3. Prisoner's dilemma of two companies' CSR.

\begin{tabular}{lccc}
\hline & \multicolumn{2}{c}{ Company 2 } \\
\cline { 3 - 4 } & & Fulfill & Not fulfilling \\
\hline \multirow{2}{*}{ Company 1 } & Fulfill & $(5,5)$ & $(1,9)$ \\
& Not fulfilling & $(9,1)$ & $(3,3)$ \\
\hline
\end{tabular}

Table 4. The payoff matrix with the government's involvement.

\begin{tabular}{cccc}
\hline & \multicolumn{2}{c}{ Company } \\
\cline { 3 - 4 } & Fulfill $(q)$ & $\begin{array}{c}\text { Not Fulfilling } \\
(1-q)\end{array}$ \\
\hline \multirow{3}{*}{ Government } & $\begin{array}{c}\text { Supervise }(p) \\
\text { Without } \\
\text { supervision }(1-p)\end{array}$ & $(8,-10)$ & $(11,-13)$ \\
\hline
\end{tabular}

We can solve that: $x=10 / 13, y=11 / 13$.

Therefore, the mixed strategy of government is $(10 / 13,3 / 13)$, the mixed strategy of company is $(11 / 13,2 / 13)$.

So, we can find that the government is willing to supervise by probability of $10 / 13$, and company willing to fulfill the social responsibility by probability of 11/13. This shows that it is still likely for government to supervise and company to undertake the social responsibility.

\section{Conclusions}

From the perspective of game theory, undertaking social responsibility for a long time is the optimal strategy of company. But in the short time, companies will choose a low-cost way to get more profits, so the construction of CSR must be based on the sustainable development. At the same time, the government should strengthen the supervision and improve the investment of social responsibility by economic, regulatory and ethical means.

It is significant for company to undertake the social responsibility, because it can implement the scientific development concept of our country and build a harmonious society. Combined with China's current situation, if we want to make enterprises much better, we should fulfill our social responsibilities. It is the key to change the idea of corporate and enable itself to realize that fulfilling their social responsibility is not only the benefit for society, but also for their rapid development. By doing it, we can fundamentally solve the problems of ful- fillment of CSR that is not in place. In the course of implementation of CSR, the government should play a watchdog role, and regulate corporate behavior through a variety of proactive measures. Throughout the course of the game, the government should grasp the performance of company's CSR and conduct mechanism innovation, as well as encourage companies to undertake their social responsibility conscientiously. While the enterprises are in the pursuit of getting more benefits, they should also have the courage to undertake the social responsibility, establish a social responsibility-oriented corporate culture and a good corporate image, improve the moral construction and transparency of the company, and strive to be good company citizens. From the long-term perspective, the more social responsibility companies undertake, the more benefits they will have. It is good for its development if companies undertake more social responsibility.

\section{Acknowledgements}

This work is supported by the National Natural Science Foundation of China under Grant 11101265 and 61075115, Innovation Program of Shanghai Education Commission under Grant 14ZZ157.

\section{REFERENCES}

[1] Y. Li and S. Wei, “The Game Analysis of CSR,” Journal of Pingdingshan University, 2011, pp. 96-101+108.

[2] D. Lu and Q. Li "Economic and Legal Analysis of CSR," 1st Edition, Legal Publishing House, Beijing, 2002.

[3] L. Zhang, X. Ma and M. Li, "CSR Evaluation System Research under the Background of Low Carbon Economy," Science and Technology Management Research, 2013, pp. 67-70.

[4] F. Wei and Y. Tang, "The Value of CSR Investment Measures-Based on Solow Model," Friends of Accounting, 2013, pp. 95-98.

[5] S. Mai, H. Kuang and X. Zhang, "The Evaluation Model and Empirical Analysis of CSR That Based on the Scientific Development," Scientific Research Management, 2013, pp. 148-154. 